\title{
COMPARABLE DIFFERENTIABILITY CHARACTERISATIONS OF TWO CLASSES OF BANACH SPACES
}

\author{
J.R. GILES
}

\begin{abstract}
We characterise Banach spaces not containing $\ell_{1}$ by a differentiability property of each equivalent norm and show that a slightly stronger differentiability property characterises Asplund spaces.
\end{abstract}

A continuous convex function $\phi$ on an open convex subset $A$ of a normed linear space $X$ is Gâteaux differentiable at $x \in A$ in the direction $y \in X$ if

$$
\phi^{\prime}(x)(y) \equiv \lim _{\lambda \rightarrow 0} \frac{\phi(x+\lambda y)-\phi(x)}{\lambda}
$$

exists, and is Gâteaux differentiable at $x$ if $\phi^{\prime}(x)(y)$ exists for all $y \in X$. Further $\phi$ is Fréchet differentiable at $x$ if the limit $\phi^{\prime}(x)(y)$ is approached uniformly for all $y \in X$, $\|y\|=1$

An $A$ splund space is a Banach space $X$ where every continuous convex function $\phi$ on an open convex subset $A$ of $X$ is Fréchet differentiable on a dense $G_{\delta}$ subset of $A$. The theory of Asplund spaces is by now well established, (see [6]). Our first interest is in the following characterisations.

Proposition 1. For a Banach space $X$, the following are equivalent.

(i) $X$ is an Asplund space,

(ii) every nonempty bounded subset $K$ of $X^{*}$ has weak* slices of arbitrarily small diameter, [6, p.31],

(iii) every continuous weak* lower semi-continuous convex function $\phi$ on an open convex subset $A$ of $X^{* *}$ is Fréchet differentiable at the points of a dense $G_{\delta}$ subset of $A,[6, \mathrm{p} .94]$,

(iv) every equivalent norm $p$ on $X$ is Fréchet differentiable at some point of $X,[6$, p.33].

We note that given a nonempty bounded subset $K$ of $X^{*}$, a weak ${ }^{*}$ slice of $K$ is a nonempty subset of $K$ of the form

$$
S(K, \widehat{x}, \delta) \equiv\{f \in K: f(x)>\sup \widehat{x}(K)-\delta\}
$$

Received 13th November, 1996

Copyright Clearance Centre, Inc. Serial-fee code: 0004-9729/97 \$A2.00+0.00. 
for some $x \in X \backslash\{0\}$ and $\delta>0$.

The study of Banach spaces not containing $\ell_{1}$ is also well established, (see [8]). However, a fascinating characterisation for such spaces was given in [7, p.422].

Proposition 2. A Banach space $X$ does not contain a subspace topologically isomorphic to $\ell_{1}$ if and only if, given $F \in X^{* *}$, every nonempty bounded subset $K$ of $X^{*}$ has weak* slices over which the oscillation of $F$ is arbitrarily small.

We recall that, given $F \in X^{* *}$ and a nonempty bounded set $K$ in $X^{*}$, the oscillation of $F$ over $K$ is

$$
\omega(F(K)) \equiv \sup \{|F(f-g)|: f, g \in K\} .
$$

Because the characterisation in Proposition 2 is comparable to that given in Proposition 1 (i) $\Longleftrightarrow$ (ii), it suggests that we investigate a differentiability characterisation comparable to Proposition 1 (i) $\Longleftrightarrow$ (iii) $\Longleftrightarrow$ (iv).

A set-valued mapping $\Phi$ from a topological space $A$ into subsets of the dual $X^{*}$ of a Banach space $X$ is said to be minimal if given any open set $U \subseteq A$ and a weak* open half-space $W$ in $X^{*}$ such that $\Phi(U) \cap W \neq \emptyset$ there exists a nonempty open set $V \subseteq U$ such that $\Phi(V) \subseteq W$. Further $\Phi$ is said to be locally bounded if for every $x \in A$ there exists a neighbourhood $U$ of $x$ such that $\Phi(U)$ is bounded in $X^{*}$.

Given a continuous convex function $\phi$ on an open convex subset $A$ of a Banach space $X$, the subdifferential of $\phi$ at $x \in A$ is the set

$$
\partial \phi(x) \equiv\left\{f \in X^{*}: f(y) \leqslant \phi_{+}(x)(y) \text { for all } y \in X\right\} .
$$

Given a separated locally convex topology $\tau$ on the dual $X^{*}$, the subdifferential mapping $x \mapsto \partial \phi(x)$ is $\tau$-upper semi-continuous at $x \in A$ if given $W$ a $\tau$-open subset of $X^{*}$ such that $\partial \phi(x) \subseteq W$ there exists a $\delta>0$ such that $\partial \phi(y) \subseteq W$ for all $y \in A$, $\|x-y\|<\delta$. The subdifferential mapping $x \mapsto \partial \phi(x)$ is a minimal weak ${ }^{*}$ cusco on $A$; that is, given $x \in A, \partial \phi(x)$ is nonempty, weak* compact and convex and the mapping is weak* upper semi-continuous and minimal on $A$. It is also locally bounded. Now $\phi$ is Gâteaux differentiable at $x \in A$ if and only if $\partial \phi(x)$ is singleton and is Fréchet differentiable at $x$ if and only if $\partial \phi(x)$ is singleton and the subdifferential mapping $x \mapsto \partial \phi(x)$ is norm upper semi-continuous at $x,[6, \mathrm{p} .19]$.

Given a continuous convex function $\phi$ on an open convex subset $A$ of a Banach space $X$ we can extend $\phi$ as a lower semi-continuous convex function $\bar{\phi}$ on $X$ by defining

$$
\bar{\phi}(x)= \begin{cases}\liminf _{y \rightarrow x} \phi(y) & \text { for } x \in \bar{A} \\ +\infty & \text { otherwise. }\end{cases}
$$

The subdifferential of $\bar{\phi}$ at $x \in A$ is the set $\partial \phi(x)$. The lower semi-continuous convex function $\phi^{*}$ on $X^{*}$, the Fenchel conjugate of $\phi$ on $A$ is defined by

$$
\phi^{*}(f)=\sup \{f(x)-\bar{\phi}(x): x \in \bar{A}\} \text {. }
$$


Now $f \in \partial \phi(x)$ if and only if $\widehat{x} \in \partial \phi^{*}(f)$. Also $\left.\phi^{* *}\right|_{\widehat{A}}=\phi,[6 . \mathbf{p}$.42].

ThEOREM 1. For a Banach space $X$, the following are equivalent.

(i) $X$ does not contain a subspace topologically isomorphic to $\ell_{1}$,

(ii) for every nonempty bounded subset $K$ of $X^{*}$, given $\varepsilon>0$ and $F \in X^{* *}$ there exists $x \in X \backslash\{0\}$ and $\delta(\varepsilon, F, x)>0$ such that

$$
\omega(F(S(K, \widehat{x}, \delta)))<\varepsilon
$$

(iii) for every continuous convex function $\phi$ on an open convex subset $A$ of $X$, given $F \in X^{* *}$ the real set-valued mapping $x \mapsto F(\partial \phi(x))$ is singlevalued and upper semi-continuous at the points of a dense $G_{\delta}$ subset $D_{F}$ of $A$.

(iv) for every continuous convex function $\phi$ on an open convex subset $A$ of $X$, given $F \in X^{* *} \backslash\{0\}, \phi^{* *}$, the second Fenchel conjugate of $\phi$ on $X^{* *}$, is Gâteaux differentiable in the direction $F$ at the points of a dense $G_{\delta}$ subset $D_{F}$ of $\widehat{A}$.

(v) for every equivalent norm $p$ on $X$, given $F \in X^{* *} \backslash\{0\}$ the norm $p^{* *}$ on $X^{* *}$ induced by $p$ is Gâteaux differentiable in the direction $F$ at some point of $\widehat{X}$.

PROOF:

(i) $\Longleftrightarrow$ (ii) is Proposition 2 .

(ii) $\Longrightarrow$ (iii) Given $F \in X^{* *}$ and $\varepsilon>0$ consider the set

$$
O_{\varepsilon} \equiv \bigcup\{\text { open } U \subseteq A: \omega(F(\partial \phi(U)))<\varepsilon\}
$$

Now $O_{\varepsilon}$ is open in $A$; we show that it is dense in $A$. Consider open $U \subseteq A$ such that $\partial \phi(U)$ is bounded. By (ii) there exists a weak* slice $S$ of $\partial \phi(U)$ such that $\omega(F(S))<\varepsilon$. Since the subdifferential mapping $x \mapsto \partial \phi(x)$ is a minimal weak* cusco on $A$, there exists a nonempty open set $V \subseteq U$ such that $\partial \phi(V) \subseteq S$. But then $\omega(F(\partial \phi(V)))<\varepsilon$. We conclude that $D_{F} \equiv \bigcap_{\varepsilon>0} O_{\varepsilon}$, the set where the mapping $x \mapsto F(\partial \phi(x))$ is singlevalued and upper semi-continuous, is a dense $G_{\delta}$ subset of $A$.

(iii) $\Longrightarrow$ (iv) Suppose that $\phi^{* *}$ is not Gâteaux differentiable in the direction $F$ at $\widehat{x}_{0} \in \widehat{A}$.

Then there exist $\widehat{f}_{0}, \mathcal{F} \in \partial \phi^{* *}\left(\widehat{x}_{0}\right), \mathcal{F} \neq \widehat{f}_{0}$ and $r>0$ such that $\left(\mathcal{F}-\widehat{f}_{0}\right)(F)>r$. Since $B\left(\hat{X}^{*}\right)$ is weak ${ }^{*}$ dense in $B\left(X^{* * *}\right)$, for each $n \in \mathbb{N}$ there exists $g_{n} \in \widehat{X}^{*}$, $\left\|g_{n}\right\| \leqslant\|\mathcal{F}\|$ such that 


$$
\left|\left(\mathcal{F}-\widehat{g}_{n}\right)(F)\right|<\frac{1}{n} \text { and }\left|\left(\mathcal{F}-\widehat{g}_{n}\right)\left(\widehat{x}_{0}\right)\right|<\frac{1}{n} \text {. }
$$

Since

$$
\mathcal{F}(F)-\mathcal{F}\left(\widehat{x}_{0}\right) \leqslant \phi^{* *}(F)-\phi^{* *}\left(\widehat{x}_{0}\right) \text { for all } F \in \widehat{A}
$$

then

$$
g_{n}(x)-g_{n}\left(x_{0}\right) \leqslant \phi(x)-\phi\left(x_{0}\right)+\frac{2}{n} \text { for all } x \in A \text {. }
$$

By the Brøndsted-Rockafeller Theorem, $\left[6\right.$, p.48] there exists $x_{n} \in A$ and $f_{n} \in \partial \phi\left(x_{n}\right)$ such that

$$
\left\|x_{0}-x_{n}\right\|<\frac{2}{\sqrt{n}} \quad \text { and } \quad\left\|g_{n}-f_{n}\right\|<\frac{1}{\sqrt{n}} \text {. }
$$

Now $\left|F\left(g_{n}-f_{0}\right)\right|>r-1 / n$ so $\left\|x_{0}-x_{n}\right\|<2 / \sqrt{n}$ but $\left|F\left(f_{n}-f_{0}\right)\right|>r-1 / n-1 / \sqrt{n}$. We conclude that the mapping $x \mapsto F(\partial \phi(x))$ cannot be both singleton and upper semi-continuous at $x_{0}$.

(iv) $\Longrightarrow(v)$ is obvious.

(v) $\Longrightarrow$ (ii) Suppose that there exists a nonempty bounded set $A$ in $X^{*}$ and $F \in X^{* *}$ and $r>0$ such that every weak* slice $S$ of $A$ has $\omega(F(S))>r$. Write $C \equiv$ $c o(A \cup(-A))$ and $K \equiv C+B\left(X^{*}\right)$. Now every weak* slice $S$ of $K$ has $\omega(F(S))>r$. The functional $p$ on $X$ defined by

$$
p(x)=\sup \{f(x): f \in K\}
$$

is an equivalent norm on $X$. Given $x \in X \backslash\{0\}$, for all $n \in \mathbb{N}$

$$
\omega\left(F\left(\left\{f \in X^{*}: f(x)>p(x)-r / 3 n\right\}\right)\right)>r
$$

so there exist $f_{n}, g_{n} \in K$ such that

$$
f_{n}(x)>p(x)-\frac{r}{3 n}, g_{n}(x)>p(x)-\frac{r}{3 n} \text { and }\left|F\left(f_{n}-g_{n}\right)\right|>r-\frac{1}{n} .
$$

Therefore for $p^{* *}$ on $X^{* *}$ where $p^{* *}(F)=\sup \{F(f): f \in K\}$ we have

$$
\begin{aligned}
p^{* *}\left(\widehat{x}+\frac{1}{n} F\right) & +p^{* *}\left(\widehat{x}-\frac{1}{n} F\right)-2 p^{* *}(\widehat{x}) \\
& \geqslant \widehat{f}_{n}\left(\widehat{x}+\frac{1}{n} F\right)+\widehat{g}_{n}\left(\widehat{x}-\frac{1}{n} F\right)-\left(f_{n}+g_{n}\right)(x)-\frac{2 r}{3 n} \\
& =\frac{1}{n} F\left(f_{n}-g_{n}\right)-\frac{2 r}{3 n} \\
& >\frac{r}{3 n}-\frac{1}{n^{2}} .
\end{aligned}
$$

Then $\quad n\left\{p^{* *}(\widehat{x}+(1 / n) F)+p^{* *}(\widehat{x}-(1 / n) F)-2 p^{* *}(\widehat{x})\right\}>r / 3-1 / n$ 
and so $p^{* *}$ is not Gâteaux differentiable at $\widehat{x}$ in the direction $F$. We conclude that if, given $F \in X^{* *} \backslash\{0\}, p^{* *}$ is Gâteaux differentiable at some $\widehat{x} \in \widehat{X}$ in the direction $F$, then (ii) holds.

We note that a similar characterisation was proved by Gilles Godefroy $[3, \mathrm{p} .8]$

It is clear that the proof of Theorem 1 (i) $\Longleftrightarrow$ (iii) can be generalised.

Corollary 1. A Banach space $X$ does not contain a subspace topologically isomorphic to $\ell_{1}$, if and only if for every locally bounded minimal set-valued mapping $\Phi$ from a Baire space $A$ into subsets of the dual $X^{*}$ with its weak ${ }^{*}$ topology, given $F \in X^{* *}$ the mapping $t \mapsto F(\Phi(t))$ is single-valued and upper semi-continuous at the points of a dense $G_{\delta}$ subset $D_{F}$ of $A$.

Proposition 1 (i) $\Longleftrightarrow$ (iv) implies that a Banach space which is not an Asplund space has an equivalent norm which is nowhere Fréchet differentiable. Similarly, Theorem $1(\mathrm{i}) \Leftrightarrow(\mathrm{v})$ implies that a Banach space which contains a subspace topologically isomorphic to $\ell_{1}$ has an equivalent norm and $F \in X^{* *} \backslash\{0\}$ such that the norm on $X^{* *}$ is nowhere Gâteaux differentiable in the direction $F$. On $\left(\ell_{1},\|\cdot\|_{1}\right)$, the norm $\|\cdot\|_{1}$ is nowhere Fréchet differentiable, $[6, p .8]$, but also the norm on $\ell_{1}^{* *}$ exhibits this other property.

Proof: The norm \|\|$_{1}$ is Gâteaux differentiable only at those points $f \equiv$ $\left\{\lambda_{1}, \lambda_{2}, \ldots, \lambda_{n}, \ldots\right\}$ where $\lambda_{n} \neq 0$ for all $n \in \mathbb{N},[6$, p.3]. So it is sufficient to consider the differentiability of the norm $\|\cdot\|$ on $\ell_{1}^{* *}$ at such points $\hat{f}$ in $\widehat{\ell}_{1}$. The norm $\|\cdot\|$ on $\ell_{1}^{* *}$ is

$$
\|\mathcal{F}\|=\|\widehat{g}\|+\left\|x^{\perp}\right\| \quad \text { where } \mathcal{F}=\widehat{g}+x^{\perp} \in \ell_{1}^{* *} \text { and } x \in c_{0} \text { and } g \in \ell_{1} .
$$

Now

$$
\begin{aligned}
\frac{\|\hat{f}+\lambda \mathcal{F}\|-\|\hat{f}\|}{\lambda} & =\frac{\left\|\hat{f}+\lambda\left(\hat{g}+x^{\perp}\right)\right\|-\|\hat{f}\|}{\lambda} \\
& =\frac{\|f+\lambda g\|-\|f\|}{\lambda}+\frac{|\lambda|\left\|x^{\perp}\right\|}{\lambda} \rightarrow\|f\|^{\prime}(g) \pm\left\|x^{\perp}\right\| \text { as } \lambda \rightarrow 0 .
\end{aligned}
$$

So we conclude that for any $x^{\perp} \in c_{0}^{\perp} \backslash\{0\}$ the norm on $\ell_{1}^{* *}$ is nowhere Gâteaux differentiable on $\widehat{\ell}_{1}$ in the direction $x^{\perp}$.

We should note that Theorem 1 (i) $\Longleftrightarrow$ (v) implies that $\ell_{\infty}$ has an equivalent norm which is nowhere Gâteaux differentiable. For if every equivalent norm on $\ell_{\infty}$ had a point of Gâteaux differentiability then the fact that weak* convergent sequences are weakly convergent in $\ell_{\infty}^{*}$ would imply that Theorem $1(\mathrm{v})$ would be satisfied. But that would contradict the fact that $\ell_{\infty}$ contains a subspace isometrically isomorphic to $\ell_{1}$. 
It is known that any Banach space $X$ which contains a subspace topologically isomorphic to $\ell_{1}$ has the property that there exists $F \in X^{* *} \backslash\{0\}$ and an equivalent norm $p$ on $X$ such that

$$
p^{* *}(\widehat{x}+F)=p^{* *}(\widehat{x})+p^{* *}(F) \text { for all } x \in X,
$$

$[1$, p.107]. So Theorem $1(v) \Longrightarrow$ (i) could be deduced from this renorming property.

Using the Bishop-Phelps Theorem, [6, p.49], it is not difficult to show that given $F \in X^{* *}$ and an equivalent norm $p$ on $X$ with closed unit ball $B_{p}(X)$, the mapping $x \mapsto F(\partial p(x))$ is single-valued and upper semi-continuous at $x \in X, p(x)=1$ if and only if given $\varepsilon>0$ there exists a $\delta(\varepsilon, F)>0$ such that $\omega\left(F\left(S\left(B_{p}^{*}(X), \widehat{x}, \delta\right)\right)\right)<\varepsilon$. So using this and the previous comment we can deduce a result similar to that given in $[1$, p.112].

Corollary 2. A Banach space $X$ does not contain a subspace topologically isomorphic to $\ell_{1}$ if and only if given $F \in X^{* *}$ and an equivalent norm $p$ on $X$ there exists a point in $B_{p}^{*}(X)$ where $F$ restricted to $B_{p}^{*}(X)$ is weak ${ }^{*}$ continuous.

It is interesting to compare the characterisations given in Theorem 1 with a generalisation of the characterisations given in Proposition 1.

THEOREM 2. For a Banach space $X$ the following are equivalent.

(i) $X$ is an Asplund space,

(ii) for every nonempty bounded subset $K$ of $X^{*}$, there exists $x \in X \backslash\{0\}$ such that, given $\varepsilon>0$ and $F \in X^{* *}$ there exists $\delta(\varepsilon, F)>0$ such that

$$
\omega(F(S(K, \widehat{x}, \delta)))<\varepsilon .
$$

(iii) for every continuous convex function $\phi$ on an open convex subset $A$ of $X$, the subdifferential mapping $x \mapsto \partial \phi(x)$ is single-valued and weak upper semi-continuous at the points of a dense $G_{\delta}$ subset of $A$,

(iv) for every continuous convex function $\phi$ on on an open convex subset $A$ of $X, \phi^{* *}$, the second Fenchel conjugate of $\phi$ on $X^{* *}$, is Gâteaux differentiable at the points of a dense $G_{\delta}$ subset of $\widehat{A}$.

(v) for every equivalent norm $p$ on $X$, the norm $p^{* *}$ on $X^{* *}$ induced by $p$ is Gâteaux differentiable at some point of $\widehat{X}$.

PROOF:

(i) $\Longrightarrow$ (ii) follows from Proposition 1 (i) $\Longrightarrow$ (ii).

(i) $\Longrightarrow$ (iii) and (iv) $\Longrightarrow$ (v) are obvious.

(iii) $\Longrightarrow$ (iv) follows as in Theorem 1 (iii) $\Longrightarrow$ (iv).

(v) $\Longrightarrow$ (ii). Suppose that there exists a nonempty bounded set $A$ in $X^{*}$ such that given $x \in X \backslash\{0\}$ there exists $F \in X^{* *}$ and $r>0$ such that every weak* slice $S$ of $A$ 
generated by $x$ has $\omega(F(S))>r$. Writing $C \equiv$ co $(A \cup(-A))$ and $K \equiv C+B\left(X^{*}\right)$ and considering the equivalent norm $p$ on $X$ defined by $p(x)=\sup \{f(x): f \in K\}$, we have as in Theorem $1(\mathrm{v}) \Longrightarrow$ (ii) that $p^{* *}$ on $X^{* *}$ where $p^{* *}(F)=\sup \{F(f): f \in K\}$, is not Gâteaux differentiable at $\widehat{x}$ in the direction $F$. We conclude that if $p^{* *}$ is Gâteaux differentiable at some point of $\widehat{X}$, then (ii) holds.

(ii) $\Longrightarrow$ (i). Consider a nonempty closed bounded convex set $K$ in $X^{*}$. From (ii) there exists $x \in X \backslash\{0\}$ such that given $\varepsilon>0$ and $F \in X^{* *}$ there exists $\delta(\varepsilon, F)>0$ such that $\omega\left(F\left(S\left(\bar{K}^{w^{*}}, \widehat{x}, \delta\right)\right)\right)<\varepsilon$. So for $C \equiv\left\{f \in \bar{K}^{w^{*}}: f(x)=\sup \widehat{x}\left(\bar{K}^{w^{*}}\right)\right\}=$ $\bigcap_{\delta>0} S\left(\bar{K}^{w^{*}}, \widehat{x}, \delta\right)$ we have $\omega(F(C))=0$ for all $F \in X^{* *}$. But this implies that $C$ is a singleton and an extreme point of $\bar{K}^{w^{*}}$. Write $C \equiv\left\{f_{0}\right\}$. However, (ii) implies that given $\varepsilon>0$ and $F \in X^{* *}$ there exists $\delta(\varepsilon, F)>0$ such that

$$
\left|F\left(f-f_{0}\right)\right|<\varepsilon \quad \text { when } f \in S\left(\bar{K}^{w^{*}}, \widehat{x}, \delta\right) .
$$

But $S\left(\bar{K}^{w^{*}}, \widehat{x}, \delta\right) \cap K \neq \emptyset$ for all $\delta>0$ and we deduce that $f_{0} \in \bar{K}^{w}=K$. Therefore $f_{0}$ is an extreme point of $K$. This is sufficient to prove that $X^{*}$ has the Krein-Milman property, [2, p.190], which in turn implies that $X$ is an Asplund space, [4].

We note that the proof (ii) $\Longrightarrow$ (i) is due to Isaac Namioka, [private communication].

We are able to use Theorem 2 to deduce the following condition for a Banach space to be Asplund.

Corollary 3. $\left[5\right.$, p.501]. A Banach space $X$ where $X^{* *} / \widehat{X}$ is separable is an Asplund space.

PRoof: If $X \supseteq \ell_{1}$ then $\ell_{1}^{* *} / \widehat{\ell}_{1}$ is topologically isomorphic to a subspace of $X^{* *} / \widehat{X}$, but then $X^{* *} / \widehat{X}$ is not separable. So $X \nsupseteq \ell_{1}$. Given an equivalent norm $p$ on $X$ and $x \in X, p_{+}^{* *^{\prime}}(\widehat{x})(F)$ is continuous in $F$ so from Theorem 1 we have that $p^{* *}$ is Gâteaux differentiable at the points of a dense $G_{\delta}$ subset $D$ of $\widehat{X}$ in all directions $X^{* *} \backslash \widehat{X}$ and since $X^{* *} \backslash \widehat{X}$ is dense in $X^{* *}$ we deduce that $p^{* *}$ is Gâteaux differentiable at each point of $D$. We conclude that $X$ is an Asplund space.

An Asplund space is also characterised by the structure of the weak* compact convex subsets in its dual, $[\boldsymbol{6}, \mathbf{p} .86]$.

Given a nonempty weak ${ }^{*}$ closed convex subset $K$ of the dual $X^{*}$ of a Banach space $X$, we say that $f \in K$ is a weak ${ }^{*}$ exposed point of $K$ if there exists an $x \in X \backslash\{0\}$ such that

$$
f(x)=\sup \widehat{x}(K)>g(x) \text { for all } g \in K, f \neq g .
$$


We say that $x$ weak $^{*}$ exposes $K$ at $f$. If, when $g_{n}(x) \rightarrow f(x)$ as $n \rightarrow \infty$ for $\left\{g_{n}\right\} \subseteq K$ we have that $\left\{g_{n}\right\}$ is norm convergent to $f$, then we say that $f$ is a weak* strongly exposed point of $K$.

PROPOSITION 3. For a Banach space $X$ the following are equivalent.

(i) $X$ is an Asplund space,

(ii) every nonempty weak* closed convex subset of $X^{*}$ is the weak* closed convex hull of its weak* strongly exposed points,

(iii) every nonempty weak ${ }^{*}$ closed convex subset of $X^{*}$ has at least one weak* strongly exposed point.

A comparable characterisation can be given for a Banach space which does not contain a subspace topologically isomorphic to $\ell_{1}$.

For a nonempty subset $A$ of the dual $X^{*}$ of a Banach space $X$ we say that, given $F \in X^{* *} \backslash\{0\}$, an element $x \in X$ weak ${ }^{*} F$-exposes $A$ if given $\varepsilon>0$ there exists $\delta>0$ such that $\omega(F(S(A, \widehat{x}, \delta)))<\varepsilon$ and in this case if $\bigcap_{\delta>0} S(A, \widehat{x}, \delta)$ is nonempty we call this set a weak $k^{*} F$-exposed face of $A$.

LEMMA. Consider a continuous positive sublinear functional $p$ on a Banach space $X$ and the set $C \equiv\{x \in X: p(x) \leqslant 1\}$. Given $F \in X^{* *} \backslash\{0\}$, the real set-valued mapping $x \mapsto F(\partial p(x))$ is single-valued and upper semi-continuous at $x_{0} \in X$ if and only if $\partial p\left(x_{0}\right)$ is a weak ${ }^{*} F$-exposed face of $C^{0} \equiv\left\{f \in X^{*}: f(x) \leqslant 1\right.$ for all $\left.x \in C\right\}$.

Proof: Suppose that given $\varepsilon>0$ there exists $\delta>0$ such that $\omega\left(F\left(S\left(C^{0}, \widehat{x}_{0}, \delta\right)\right)\right)$ $<\varepsilon$. Recall that $\partial p(x) \subseteq C^{0}$ for all $x \in X$. Now

$$
\partial p\left(x_{0}\right) \subseteq W \equiv\left\{f \in X^{*}: f\left(x_{0}\right)>p\left(x_{0}\right)-\delta\right\}
$$

Since the subdifferential mapping $x \mapsto \partial p(x)$ is weak* upper semi-continuous at $x_{0}$ there exists a neighbourhood $N$ of $x_{0}$ such that

$$
\partial p(x) \subseteq W \cap C^{0}=S\left(C^{0}, \widehat{x}_{0}, \delta\right) \text { for all } x \in N
$$

So then $\omega(F(\partial p(N)))<\varepsilon$ implying that the mapping $x \mapsto F\left(\partial p\left(x_{0}\right)\right)$ is single-valued and upper semi-continuous at $x_{0}$.

For the converse, we may assume that $\|F\|=1$. Consider the mapping $x \mapsto$ $F(\partial p(x))$ single-valued at $x_{0}$. Suppose that there exists a sequence $\left\{f_{n}\right\}$ in $C^{0}$ and $r>0$ such that

but

$$
f_{n}\left(x_{0}\right) \rightarrow \partial p\left(x_{0}\right)\left(x_{0}\right)=p\left(x_{0}\right) \text { as } n \rightarrow \infty
$$


Then there exists a subsequence $\left\{f_{n_{k}}\right\}$ such that

$$
f_{n_{k}}\left(x-x_{0}\right) \leqslant p(x)-p\left(x_{0}\right)+\frac{1}{k^{2}} \text { for all } x \in X \text { and } k \in \mathbb{N} \text {. }
$$

Then by the Brøndsted-Rockafeller Theorem, $[6, \mathrm{p} .48]$, for each $k \in \mathbb{N}$ there exist $x_{k} \in X$ and $f_{k} \in \partial p\left(x_{k}\right)$ such that $\left\|x_{k}-x_{0}\right\| \leqslant 1 / k$ and $\left\|f_{n_{k}}-f_{k}\right\|<1 / k$. So $x_{k} \rightarrow x_{0}$ as $k \rightarrow \infty$ but

$$
F\left(f_{k}-\partial p\left(x_{0}\right)\right)>\frac{r}{2} \text { for all } k>\frac{2}{r}
$$

and we conclude that the mapping $x \mapsto F(\partial p(x))$ is not upper semi-continuous at $x_{0}$.

Theorem 3. For a Banach space $X$, the following are equivalent.

(i) $X$ does not contain a subspace topologically isomorphic to $\ell_{1}$,

(ii) given $F \in X^{* *} \backslash\{0\}$, every nonempty weak* compact convex subset of $X^{*}$ is the weak ${ }^{*}$ closed convex hull of its weak ${ }^{*} F$-exposed faces,

(iii) given $F \in X^{* *} \backslash\{0\}$, every nonempty weak* compact convex subset of $X^{*}$ has at least one weak ${ }^{*} F$-exposed face.

Proof:

(iii) $\Rightarrow$ (i). Given any nonempty bounded set $K$ in $X^{*}$, the weak ${ }^{*}$ closed convex hull of $K$ has weak* $F$-exposed faces. So $K$ has weak* slices over which the oscillation of $F$ is arbitrarily small. By Proposition 2 we have that $X$ does not contain a subspace topologically isomorphic to $\ell_{1}$.

(i) $\Rightarrow$ (ii). Consider $A$ a nonempty weak* compact convex subset of $X^{*}$. We may assume that $0 \in A$ and we define

$$
p(x)=\sup \{f(x): x \in A\} \equiv M(x, A)
$$

Then $p$ is a continuous positive sublinear functional on $X$ and is the gauge of $C \equiv$ $\{x \in X: p(x) \leqslant 1\}$ and $C^{0}=A$. Consider $K$, the weak* closed convex hull of the weak* $F$-exposed faces of $A$, and suppose that $K \neq A$. Then there exists $x \in X$ such that $M(x, K)<M(x, A)$. Since both $M(x, K)$ and $M(x, A)$ are continuous on $X$ then $\{x \in X: M(x, K)<M(x, A)\}$ is open in $X$. By Theorem 1 (i) $\Leftrightarrow$ (iii), this set contains a point $x_{0}$ where the real set-valued mapping $x \mapsto F(\partial p(x))$ is single-valued and upper semi-continuous. Then by the Lemma, $\partial p\left(x_{0}\right)$ is a weak ${ }^{*} F$-exposed face of $C^{0}=A$. Then $\partial p\left(x_{0}\right)\left(x_{0}\right)=M\left(x_{0}, A\right)$. But this contradicts our supposition about $K$.

(ii) $\Rightarrow$ (iii) is obvious. 
Again it is interesting to compare the characterisation given in Theorem 3 with a generalisation of the characterisation given in Proposition 3.

For a nonempty weak* closed convex subset $K$ of the dual $X^{*}$ of a Banach space $X$ and $x \in X$ where $x$ weak $^{*}$ exposes $K$ at $f \in K$, we say that $f$ is a weak ${ }^{*}$ weak exposed point of $K$ if given $\varepsilon>0$ and $F \in X^{* *} \backslash\{0\}$ there exists a $\delta(\varepsilon, F)>0$ such that $\omega(F(S(A, \widehat{x}, \delta)))<\varepsilon$. Clearly $f \in K$ is a weak* weak exposed point of $K$ if and only if $x$ weak $^{*} F$ exposes $K$ at $f$ for every $F \in X^{* *}$.

From Theorem 2 and the Lemma we have the following characterisation.

TheOREM 4. For a Banach space $X$ the following are equivalent.

(i) $X$ is an Asplund space,

(ii) every nonempty weak* compact convex subset of $X^{*}$ is the weak ${ }^{*}$ closed convex hull of its weak* weak exposed points,

(iii) every nonempty weak* compact convex subset of $X^{*}$ has at least one weak* weak exposed point.

\section{REFERENCES}

[1] R. Deville, G. Godefroy and V. Zizler, Smoothness and renormings in Banach spaces, Pitman monographs 64 (Longman, Essex, New York, 1993).

[2] J. Diestel and J.J. Uhl, Vector measures, Math. Surveys 15 (American Mathematical Society, Providence, R.I., 1977).

[3] G. Godefroy, 'Metric characterisation of first Baire class linear forms and octahedral norms', Studia Math. 95 (1989), 1-15.

[4] R.E. Huff and P.D. Morris, 'Dual spaces with the Krein-Milman property have the Radon-Nikodym property', Proc. Amer. Math. Soc. 49 (1975), 104-108.

[5] T-H Kuo, 'On conjugate Banach spaces with the Radon-Nikodym Property', Pacific $J$. Math. 59 (1975), 497-503.

[6] R.R. Phelps, Convex functions, monotone operators and differentiability, Lecture Notes in Math. 1364, (2nd ed.) (Springer-Verlag, Berlin, Heidelberg, New York, 1993).

[7] E. Saab and P. Saab, 'A dual geometric characterization of Banach spaces not containing $\ell_{1}$ ', Pacific J. Math. 105 (1983), 415-425.

[8] D. van Dulst, Characterizations of Banach spaces not containing $\ell_{1}$ (Centrum voor Wiskunde en Informatica Tract 59, Amsterdam, 1989).

Department of Mathematics

The University of Newcastle

New South Wales 2308 Australia 